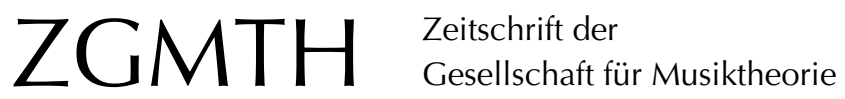

Breyer, Knud (2012): Clemens Kühn / John Leigh (Hgg.), Systeme der Musiktheorie, Dresden: Sandstein 2009. ZGMTH 9/1, 149-154. https://doi.org/10.31751/664 C 2012 Knud Breyer

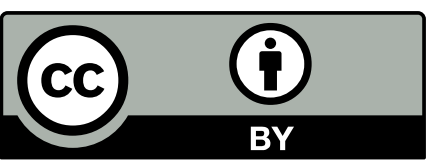

Dieser Text erscheint im Open Access und ist lizenziert unter einer Creative Commons Namensnennung 4.0 International Lizenz.

This is an open access article licensed under a

Creative Commons Attribution 4.0 International License.

veröffentlicht / first published: 26/11/2012

zuletzt geändert / last updated: 04/09/2014 


\section{Clemens Kühn / John Leigh (Hgg.), Systeme der Musiktheorie, Dresden: Sandstein 2009}

In seinem knappen Vorwort zu dem von ihm gemeinsam mit John Leigh herausgegebenen Band Systeme der Musiktheorie spricht Clemens Kühn das ungewollte Dilemma dieses Buchprojektes aus. Angesicht »einer musiktheoretischen Landschaft, die sich ebenso animierend vielfältig wie fast unübersichtlich aufgesplittert hat", läge, so Kühn, »das Thema sSysteme der Musiktheorieく [...] gegenwärtig im Grunde auf der Hand«. Und so versuche der »Band als eine Art Vergewisserung [...] gebündelt darzustellen, was sich - inmitten eines erfrischenden musiktheoretischen Pluralismus - als tragende Stützen ausmachen lässt«. Gemeint seien aber, so Kühn, »natürlich nicht Systeme alten Schlages, die Musik in ein Korsett dogmatischer Verbindlichkeiten zwangen«. Vielmehr sei "System« hier zu verstehen als »ein Synonym für verschiedene, bedeutsame Denkansätze, deren Ideen, Anliegen und Methoden sich hinreichend geschlossen beschreiben lassen« (5).

Freilich erhebt sich die Frage, ob nicht gerade die skeptische Haltung gegenüber dem herkömmlichen Systembegriff erst zum Verlust tragender Stützen geführt hat. Blickt man in das Inhaltsverzeichnis, fällt jedenfalls auf, dass zum einen die musiktheoretischen Systeme "alten Schlages" in dem schmalen, gerade einmal 131 Seiten umfassenden Band eine bedeutende Rolle spielen, so beispielsweise die Funktionstheorie Hugo Riemanns und die Schichtenlehre Heinrich Schenkers, und zum anderen vor allem Systeme respektive Denkansätze zur Sprache kommen, die schon einige Generationen, wenn nicht gar hunderte von Jahren alt sind. Der Band steckt also nicht nur einen weiten Horizont von der barocken Figurenlehre bis zur spitch class set theory، $a b$, sondern legt auch die Vermutung nahe, dass das aktuelle musiktheoretische Denken entweder primär historisch ausgerichtet ist oder aber die Systeme "alten Schlages" - ungeachtet aller Systemkritik - eben doch von besonderer metatheoretischer Qualität sind und nach wie vor als wesentlicher Ausgangsund Bezugspunkt taugen. Vor diesem Hintergrund erstaunt, dass nur ein einziger Artikel den Begriff "System« im Titel trägt - Christian Thoraus Beitrag »Interagierende Systeme - Überlegungen zu einem zeichentheoretischen Rahmen musikalischer Analyse«. Aber auch die Abschlussdiskussion der beiden Herausgeber über die Frage "Was ist ,Form»?» berührt Grundlegendes (nicht nur) musiktheoretischer Systeme. Das Gespräch ist idealtypisch dialektisch angelegt und führt zum wissenschaftstheoretischen Kern der Systemdebatte. John Leigh vertritt hierbei ein Wissenschaftsverständnis, das die »Herauskristallisierung des Elementarsten [fordert], woraus die Individualisierung erwachsen kann« (124). Leider findet Leighs Plädoyer für einen umfassenden interdisziplinären Ansatz, der zum Erfassen von Universalien und "generellen Codes« (123) aktuelle physiologische, physikalische und anthropologische Forschung mit einbezieht, im Band keinen Niederschlag. So bleibt offen, ob die Diskussion von Befunden aus der Quantenphysik oder Gen- und Hirnforschung für die Musiktheorie im Hinblick auf Formgestaltung und Formwahrnehmung überhaupt relevant ist. Nicht zu Unrecht jedenfalls verweist Kühn auf zwei Gefahren, nämlich erstens die der Beliebigkeit bei der Verbindung zwischen dem Generellen und dem Besonderen, wenn diese lediglich metaphorisch und nicht logisch begründet ist, und zweitens jene der theoretischen Versteinerung in »normativen Gefängnissen«, in welche die 
Suche nach dem Elementaren und Universellen führe. Beispiele für einen solchen »Vorrang" des »Normative[n] « gäben etwa die Kompositionslehren von Adolf Bernhard Marx und Hugo Riemann (125). Kühn plädiert daher für ein Vorgehen, das vom Besonderen sowie historisch Bedingten ausgeht, um im Anschluss durch die Gewinnung von Modellen, Topoi und Archetypen zu Generalisierungen zu kommen, durch die das Individuelle greifbar werden soll. Dagegen wendet wiederum Leigh ein, diese Vorgehensweise setze "ein Labyrinth von Prämissen voraus" und schließe »musikalische Formen aus, die diesen nicht konform sind" (124).

Aus diesem Konflikt scheint zunächst Christian Thorau mit seinem Rekurs auf die Semiotik einen Ausweg zu weisen. Nach einer knappen und anschaulichen Diskussion semiotischer Begrifflichkeiten und Paradigmen entwickelt Thorau in Anlehnung an Nelson Goodmans Symboltheorie, die auf die Besonderheit der ästhetischen Kommunikationsform in zeichentheoretischer Hinsicht abhebt, am Modellfall von Robert Schumanns Vogel als Prophet ein hermeneutisches Modell, das auf der Interaktion von Implikationssystemen beruht. Das Stück erweist sich dabei als ideales Beispiel für »Musik als offene Metapher " (78): Hier interagieren nicht nur drei Implikationssysteme miteinander - erstens die »buchstäbliche Exemplifikation « auf der Ebene der Struktur, zweitens die "metaphorische Exemplifikation « auf der Ebene des Ausdrucks und drittens die »Denotation « im Titel (80) -, sondern es ist zudem der metapherntypische semiotische »Konflikt zwischen Ähnlichkeit und Differenz« im Rätselcharakter des Titels als "passender Widerspruch" thematisiert (81). Die Stimmigkeit des Beispiels verdeckt aber auch die möglichen Probleme des zeichentheoretischen Modells. Denn wie zu verfahren ist, wenn die "analytisch-semantische Zuordnung« (82) gestört wird, bleibt unklar. Zu bedenken wäre jedenfalls, dass Schumann nach eigenen Angaben bei den Kinderszenen die Titel erst nachträglich gesetzt hat. Und ob es für die Selbstverständlichkeit, dass "wissenschaftlich generiertes Wissen [...] in diesem
Fall z.B. musikhistorische Erkenntnisse aus der Schumann-Forschung [...] die Implikationssysteme im Sinne einer historischen und semiotischen Angemessenheit der Analyse mitstrukturieren « (83), eines zeichentheoretischen Überbaus überhaupt bedarf, ist fraglich.

Thoraus Befunde lassen sich über seinen Modellfall hinaus ganz grundsätzlich für die musiktheoretische Diskussion fruchtbar machen, denn auch hier wird mit fixierbaren Codes und offenen Interaktionsgebilden, mit Denotation und metaphorischen Explikationssystemen operiert. Dass »das musikinterne Spannungsverhältnis zwischen Regel und Abweichung, Norm und Individuation [...] Übertragungen [...] involviert [...], die Interaktion und Konflikt erzeugen" (ebd.), kann auch in der Musiktheorie als beständiges Interagieren von Implikationssystemen beobachtet werden. Paradigmatisch lässt sich dies am Beispiel der Figurenlehre verdeutlichen. Ihr sind im Band gleich zwei Artikel gewidmet, die ein interessantes Spannungsverhältnis ausbilden, leider ohne dass durch die Platzierung im Band ihre Komplementarität angemessen zur Geltung käme. Johannes Korndörfers Überzeugung, "wie bereichernd die Analyse eines Musikstücks anhand eines historischen 'Theoriesystems « sei und wie dadurch »wichtigen Entwicklungen der Musikgeschichte auf die Spur [ge]kommen« werden könne (85), gilt nicht nur für ein zeitgenössisches Verhältnis von Theorie und Praxis, dem sich sein eigener Artikel »Christoph Bernhards Figurenlehre im Werk von Heinrich Schütz« widmet. Dass Korndörfer in Schütz' Historia der freuden- und gnadenreichen Geburt Gottes und Marien Sohnes, Jesu Christi SWV 435 von 1664 den zahlreichen Gebrauch jener satztechnischen Figuren feststellen kann, die dessen Schüler Christoph Bernhard in seiner Kompositionslehre Tractatus compositionis augmentatus vier Jahre zuvor systematisierte, darf als ein kaum überraschender Befund gelten. Demgegenüber ist die von Hartmut Fladt in seinem Beitrag »Modell - Topos - Figur. Individualisierte Satztechniken in Wagners ITristan und Isolde«" verfolgte These einer Präsenz "von topologischen Traditionen« (20) gerade 
dort, wo das Satzbild größte Eigenständigkeit vorgibt, höchst bemerkenswert.

Auch in anderer Hinsicht prägen die beiden Artikel eine erhellende Gegensätzlichkeit aus. Wie Korndörfer richtig ausführt, ging es Bernhard in seiner Figurenlehre darum, die aus der italienischen Opernpraxis kommende neuartige Dissonanzbehandlung ästhetisch zu rechtfertigen und dem herkömmlichen Kontrapunktverständnis zur Seite zu stellen. Korndörfer kann denn auch einige Beispiele des textausdeutenden Gebrauchs von Figuren bei Schütz zeigen, muss aber letztlich einräumen, Schütz verwende die Figuren weitaus häufiger »ohne jegliche semantische Bedeutung", wobei als Besonderheit die Kombination mehrerer Figuren hervorsteche (92). Körndorfers Einschätzung zu folgen, hieße, Schütz habe das System des Denotationszusammenhangs zwischen gattungsmäßiger Zweckbestimmung, Textbedeutung, rhetorischem Etikett und kontrapunktischem Regelverstoß aufgebrochen und den neuen Kontrapunkt zu einem eigenständigen und nicht mehr legitimationsbedürftigen technischen Verfahren erhoben. Dass aber das Verhältnis zwischen Lizenz und Semantik die Zeiten überdauert habe, ist gerade die zentrale Behauptung Hartmut Fladts, demzufolge viele der »in der Literatur geheimnisumwitterte[n] « Akkorde und Akkordverbindungen in Wagners Tristan und Isolde als "verblüffend konstruktive Konsequenz aus den selbstgesetzten Prämissen« (25) erscheinen, wenn man sie scheinbar ahistorisch auf kontrapunktische Modelle wie den Gymel oder die gestische Qualität rhetorischer Figuren, wie Interrogatio, Exclamtio, Graduatio, Climax, Auxesis, Aposiopesis und Passus duriusculus zurückprojiziert. Für Fladt verbinden sich so bei Wagner das moderne System der Tonalität, bis ins Mittelalter zurückreichende Modelle und die Ausdruckskraft der Figurenlehre zu einer hochindividuellen musikalischen Sprache.

Während sich also in der Kompositionspraxis - folgt man Fladt - verschiedene systematische und historisch konnotierte Ansätze erhalten und durchdrungen haben, kam es in der Theoriegeschichte zu einer kategorialen
Trennung von vormals Zusammengehörigem, wie die »Aufspaltung des Generalbasses in 'Wissenschaft und 'Praxis`« zeigt (10). Als deren Ursache macht Ludwig Holtmeier in seinem Artikel »Zum Tonalitätsbegriff der Oktavregel «, die »Erfindung der Oktavregel um 1700 herum« (ebd.) aus, mit der der "Generalbass zur „Harmonielehre` im modernen Sinne» (11). Während das traditionelle Generalbassdenken auf verschiedenen kontrapunktischen Modellen und Topoi beruhe, also die regelgerechte Intervallprogression in Klauseln formuliere und diese zu größeren (horizontalen) Kadenzzusammenhängen ausgebaut habe, die benenn- und erlernbar waren, harmonisiere die Oktavregel die auf- und absteigende diatonische Skala in spezifischer Weise. Die feste Koppelung von Klangqualität und Stufe führt laut Holtmeier "zu einer bislang unbekannten Vertikalisierung des harmonischen Diskurses« (ebd.). Die Oktavregel lasse sich historisch aus der Doppia-Kadenz herleiten, in der sich dialektisch »ralte Intervallprogression und sneue c Corellische Kadenzharmonik" verschränkten (16). Da letztere auf dem Prinzip der Abfolge von Spannungs- und Ruheakkorden basiere, trete "an die Stelle drei- und viertöniger Klauselzusammenhänge eine weitgehend entrhythmisierte ıZweierbeziehung، von Akkorden « (11). Zwar erkennt Holtmeier die Bedeutung dieses Paradigmenwechsels, der eine »Emanzipation der Klänge« (15) mit sich bringe, einerseits ausdrücklich an. Andererseits polemisiert er aber bisweilen von der Warte der Partimento-Tradition aus gegen die seines Erachtens problematische Seite dieser Entwicklung. So hebt er bei Rameaus Theorie der sbasse fondamentale`, die hier anknüpft, vor allem die systematische Widersprüchlichkeit und den Verlust eines differenzierteren Dissonanzbegriffs hervor, wie überhaupt bei Rameau nach Holtmeier die "Tendenz zur formalistischen Abstraktion und sesprit du système`, $[. .$.$] in den späten Schriften oft abstru-$ se Formen annimmt (18). Und »die deutsche bürgerliche Harmonielehretradition« (9) bezichtigt er, die Partimento- und Generalbasslehrbücher insbesondere deshalb nicht ernst genommen zu haben, "weil sie meist nur aus 
Noten [...] bestehen« (6). Schenker mit seinen Diagrammdarstellungen scheint Holtmeier diesem geistigen Umfeld offenbar nicht zuzurechnen. Nun mag es zwar sein, dass die Riemann-Tradition ein »undifferenziertes und ausgesprochen einseitiges Verständnis von ,Generalbass " hatte und man "geradezu manisch auf die Ziffernschrift fixiert" war; dass es aber nur darum ging, »Türmchen über den betreffenden Bassnoten « zu bauen und »harmonische Progression [...] immer den Sprung von Akkord zu Akkord" bedeutete (ebd.), scheint mit Blick auf Riemanns Funktionsbegriff dann doch eine gewagte Behauptung. Vielmehr begegnet hier ein neues Theorieverständnis, das sich nicht mehr mit der Kontingenz von Modellen und Topoi zufriedengeben mochte, sondern, wie bereits die im Band völlig ausgeblendete mittelalterlich und frühneuzeitliche Musiktheorie, nach den objektiven Prinzipien und der allgemeinen Natur der Musik fragte. Schon bei Rameau lässt sich ein Theorieansatz ausmachen, der, statt primär auf den didaktischen Nutzen einer Kompositionslehre abzuheben, Musiktheorie als Wissenschaft begreift.

Wie wirkmächtig vor diesem Hintergrund dessen Fundamentalbasslehre war, zeigt die Folgediskussion im 19. und 20. Jahrhundert. In seinem wisssenschaftstheoretisch ausgerichteten Artikel rekapituliert Bernd Redmann die Einbettung der Musiktheorie in die allgemeine wissenschaftsgeschichtliche Entwicklung, die in der zweiten Hälfte des 19. Jahrhunderts vor allem einem vom Neukantianismus inspirierten naturwissenschaftlichen Wissenschaftsideal verpflichtet war, das sich vornehmlich der Suche nach »naturgegeben, gemeinsamen Codes" (57) sowie nach einer auf »normative[n], kontextunabhängige[n], [...] zeitenthobene[n] [...] Prinzipien « (57) beruhenden "Universallogik" verschrieben habe (58). Dass ein solcher Ansatz, der aus einfachen Grundsätzen mit der Maßgabe systemimmanenter Stimmigkeit weitreichende Konsequenzen mit normativem Anspruch zieht, leicht in Konflikt mit der vielfältigen empirischen Wirklichkeit eines Gegenstands gerät, der eben nicht naturhaft gegeben, sondern der historischen Wandlung unter Veränderung der kompositionstechnischen Grundlagen unterworfen ist, wurde auch von den Protagonisten der Funktionstheorie erkannt. So schränkte bereits Riemann unter dem Eindruck historischer und ethnologischer Forschung die Extensionalität seiner Theorie in seinen späteren Schriften ein. Gleichwohl betont Redmann, dass selbst in Bezug auf die stonale Klangsyntax« (63), dort also, wo die Funktionstheorie ihr Terrain hat, sie wegen ihres "Deutbarkeits-Zwang[s] « (62), aber auch aufgrund der »häufig [...] auf die ,Vertikale` eingeengten Perspektive (65) rasch an ihre interpretatorischen Grenzen stößt, beispielsweise, wenn sie es mit historischen Topoi und Modellen, erweiterter Tonalität, auskomponierten Stufen und Stimmführungsklängen zu tun bekommt; ganz zu schweigen von der grundsätzlichen Problematik, dass die Funktionstheorie den »Einzelklänge[n] [...] die Funktionen [...] unabhängig von ihrer Position in einem Klanggefüge [...] >diktiert« (65). Außerdem bleibe fraglich, »wie überhaupt [...] zu entscheiden [...] ist, welche Akkorde in einem Werkkontext als Funktionsklänge gelten sollen?« (65). Dennoch sind der hermeneutische Wert der Funktionstheorie und damit ihre heuristische Zukunftsfähigkeit laut Redmann nicht hoch genug einzuschätzen. „Unleugbar beruht der sorganische Aufbau insbesondere großangelegter Formen auf mehr- oder sogar vielfachen harmonischen Schichtungen: Und kein anderes System der Klanganalyse«sei, so Redmann, »in der Lage, den integrativen Charakter dieser harmonischen Schichtungen und die Funktionsweise des Wechsels zwischen den Schichtenbezügen so präzise und plastisch zu fassen, wie die Funktionstheorie« (68). Schenker-Anhänger würden hier wohl Einspruch einlegen.

Dass diese Form von sPräzision und Plastizität einen hohen Preis haben kann, zeigt Konstanze Franke in ihrem Beitrag »Modulationstheorie und musikalische Wirklichkeit». Ihre zahlreichen Beispiele belegen, dass die Grundannahme Riemanns, Modulationsvorgänge ließen sich an »Umdeutungsakkorden« (107) festmachen und klassifizieren, empirisch nur bedingt haltbar ist. Allerdings beschäftigt 
sich Franke auch weniger mit Modulationstheorie als vielmehr mit Modulations- bzw. Harmonielehre, also mit der in didaktischer Absicht notwendigerweise verkürzenden $\mathrm{Zu}$ richtung einer umfassenden Theorie auf prägnante Lehrsätze, idealtypische Abstraktionen und schulmeisterliche Empfehlungen. Dergestalt ist eine seriöse Diskussion freilich nur schwer denkbar.

Ohnehin zeigt sich im Band als ein kontinuierliches Problem der "musiktheoretische[n] Landschaft« (5) die Unentschiedenheit darüber, ob sich die Musiktheorie vor allem als Wissenschaft oder als didaktische Disziplin für den Satzlehre- und Kompositionsunterricht versteht. Während im Fall von Funktionstheorie und Harmonielehre beide Seiten der Medaille betrachtet und gegeneinander abgewogen werden können, hat es im Bereich der Instrumentation, folgt man Edith Metzners historischem Abriss der Instrumentationslehre von Berlioz bis Sevsay in ihrem Beitrag "Wege zu einer neuen Instrumentationslehre« Ansätze zu einer Systematisierung nur im Rahmen didaktischer Konzepte gegeben, aber keine eigentliche Theorie der Instrumentation. Denn soll die Instrumentation gleichwertig in die Strukturanalyse einfließen, wie Metzner dies zu Recht fordert, dann müsste eine andere Darstellung gefunden werden als das Verweisen auf den Instrumentenvorsatz oder die verbale Wiedergabe eines Höreindrucks. Weiterführende Gedanken - interessant wäre beispielsweise die Erörterung einer möglichen Einbeziehung der Spektralanalyse - bleibt Metzner allerdings schuldig.

Wie problematisch jedoch der Versuch sein kann, einen streng naturwissenschaftlich ausgerichteten Ansatz für die herkömmliche Strukturanalyse fruchtbar machen zu wollen, zeigt Stephan Lewandowskis Beitrag "Zusammenhang durch Abstraktion. Die pitch class set theory nach Allen Forte« (93 ff.). Fortes Theorie erlaubt mithilfe einer mengentheoretischen Methode die Klassifizierung von diastematischen Konstellationen. Die strenge Formalisierung ist aber, sobald sie ihre Domäne der objektiven Kategorisierung von (insbesondere atonalen) Akkorden überschreitet und sich in das Feld der musikalischen Analyse begibt, auf eine Vielzahl von kontingenten, nicht durch das System erfassbaren Vorannahmen angewiesen. Welche Töne jeweils einem spitch class set zuzuordnen sind, scheint ganz beliebig, wie die Material-»Analyse « Jan Maegaards von Arnold Schönbergs op. 23,2 zeigt, die Lewandowski in spitch class sets، übersetzt. Die Zuordnung springt zwischen Horizontaler und Vertikaler und trennt rechte und linke Hand, als ob es sich um separate Tonsätze handele. Des Weiteren ist der Abstraktionsgrad bei der Umformung zu mathematisch beschriebenen Mengen hoch. Fortes Theorie blendet wesentliche Aspekte des Tonsatzes wie Höhe, Lage und sogar Abfolge der Töne einfach aus. Wenn dann noch, wie im vorliegenden Beispiel, mit Klassen von bis zu zehn Elementen operiert wird, lässt sich ein umfassendes Beziehungsgeflecht zwar leicht suggerieren, dessen Bezug zur konkreten Struktur des musikalischen Gegenstands bleibt aber vage.

Musiktheoretische Systeme jedoch, die antreten, die musikalische Struktur lückenlos zu erfassen, sehen sich dem Vorwurf ausgesetzt, totalitär zu sein. In seiner tiefgründigen Darstellung der Schichtentheorie Schenkers, tritt Oliver Schwab-Felisch dieser Kritik zwar überzeugend entgegen, allerdings, indem er weniger mit Schenker selbst argumentiert, als vielmehr mit dem derzeitigen Reflexionsstand des Ansatzes. Während man anhand der von Schwab-Felisch zitierten Äußerungen nämlich den Eindruck gewinnen kann, dass Schenker den von seinen Kritikern als problematisch empfundenen methodischen Monismus bei der Suche nach der »objektiven Wahrheit der Werke« (45) für den Ausweis von Überzeugungskraft hält, hebt Schwab-Felisch beim Verhältnis der vom allgemeinen Ursatz bis zur individuellen Ausführung reichenden Schichten weniger auf die von Schenker betonten Kategorien Logik, Kausalität und Hierarchisierung $a b$, sondern stellt eher das Moment der Interaktion und des Netzwerkscharakters sowie die Pragmatik der graphischen Darstellung und die potentielle Offenheit der Analyse als Interpretation heraus. 
In seinem Postskriptum geht Schwab-Felisch scharf mit den Kritikern der immanenten Strukturanalyse ins Gericht. Inzwischen, so Schwab-Felisch, ginge ihr Dogmatismusvorwurf sogar schon so weit nicht nur zu bemängeln, dass »Theoretiker dazu neigen, Modelle so zu konstruieren, dass sie für universal gültig und lückenlos gehalten werden können « (49), vielmehr stießen »sich manche bereits daran, »dass Theoretiker dazu neigen, Modelle zu konstruieren « (50) und versuchten im Gegenzug Theorie in historische Betrachtung zu überführen (ebd.). Zwar seien "Geschichte und Theorie [...] einander Hilfswissenschaften: Geschichte nutzt theorieerzeugte Kategorien, Theorie (theorie-) geschichtliches Wissen« (ebd.). Nicht zu übersehen sei aber die kategoriale Differenz. "Geschichtsschreibung geht vom Gegenstand, Musiktheorie dagegen von Aspekten aus. Geschichte sieht das Einzelne als Schnittpunkt heterogener Bestimmungen. Musiktheorie dagegen bildet allgemeine Kategorien und bestimmt, wie das Einzelne ihnen zu subsumieren ist» (ebd.). Schwab-Felisch' Befund, die »deutschsprachige Musiktheorie folgt gegenwärtig eher dem Paradigma des Gegenstands als dem des Aspekts« (ebd.), wird auch durch den vorliegenden Band bestätigt. So geht es nach Redmann darum, das Besondere in das »offene, freie Feld der Interpretation, der satztechnischen Kontextualisierung, der Vernetzung [von] Theorieansätzen« (69) zu stellen, und Kühn schlägt vor, mit einer beliebigen Fülle von sehr allgemeinen und unspezifischen Metaphern zu arbeiten, etwa »Prinzipien und Ideen des Formens: [z. B.] wiederholen, variieren, kontrastieren, aufgreifen, weiterführen, unterbrechen« (125). Aber, so Schwab-Felisch, "Relativismus ist [...] eine schwache, weil vortheoretische Strategie. Sie fördert die Disziplin Musiktheorie nicht, sondern behindert sie. Musikgeschichte, Geschichte der Musiktheorie und Analyse nutzen und reflektieren gegebene Theorien. Theoriebildung aber, die Aufgabe von Musiktheorie, verlangt Positionierung, nicht Relativierung « (50). Diese Diskussion hätte man sich in einem Band Systeme der Musiktheorie gewünscht. Die vorliegende Aufsatzsammlung bedarf also der Ergänzung durch einen Folgeband, der eher dem Paradigma des 'Aspekts` folgt und vor allem den Systembegriff präziser fasst. Denn ob es sich bei der spitch class set theory، um ein genuin musiktheoretisches System und bei der Figurenlehre oder den Generalbassmodellen überhaupt um Systeme handelt, müsste erst noch erörtert werden. Einstweilen bietet der Band nicht mehr als einen brauchbaren Einstieg in das Thema, wobei in die verschiedenen Ansätze allgemeinverständlich eingeführt wird. Wünschenswert wäre ein sorgfältigeres Lektorat gewesen, das die zahlreichen Druck- und Formatierungsfehler, die falsche Generalbassbezeichnung (15, Notenbeispiel 7) und den chronologischen Lapsus, Musikbeispiele von Brahms in der Instrumentationslehre Berlioz' von 1844 zu vermissen (113), beseitigt hätte.

Knud Breyer 